\title{
Role of Continuous Glucose Monitoring in Insulin-Requiring Patients with Diabetes
}

\author{
John B. Welsh, MD, PhD
}

\section{Introduction}

$\mathbf{T}$ Te alluringly simple premise of continuous glucose monitoring (CGM) is that real-time glucose concentration data can be leveraged into better in-the-moment diabetes management decisions that culminate in better outcomes. Retrospective reviews of the same data can lead to pattern discoveries that motivate changes to diet and exercise routines or to the amount and timing of insulin with respect to meals. ${ }^{1}$

Because of insulin's narrow therapeutic index, CGM is particularly appropriate for people with type 1 diabetes (T1D) and for people with type 2 diabetes (T2D) who require it. CGM alarms are potentially lifesaving for people at risk for nocturnal hypoglycemia and any time for people with impaired awareness of hypoglycemia, and the ability of CGM data to be distributed in real time is critical for those involved in the support of others with diabetes. Insulin-induced hypoglycemia continues to limit therapy intensification efforts in diabetes and contributes to many population-based A1C goals not being met. With real-time CGM, the association between lower $\mathrm{A} 1 \mathrm{C}$ values and higher rates of hypoglycemia is weakened and may even be abolished. ${ }^{2,3}$ The information and insights provided by CGM are allowing many people with insulin-requiring diabetes to set and reach more aggressive glycemic goals.

\section{Recent Advances in CGM}

In the decade since publication of the JDRF CGM study, ${ }^{4}$ CGM accuracy has improved ${ }^{5}$ and its use has increased dramatically among youth and young adults with T1D, ${ }^{6}$ but is still low. As of early 2017, $\sim 24 \%$ of patients in the T1D Exchange Clinic Registry were using CGM. ${ }^{7}$ Broadening of inclusion criteria and improvements in CGM performance and usability have and will continue to fuel increases in patient adoption. A real-time CGM system (Dexcom G5 ${ }^{\circledR}$
Mobile) can be used for insulin dosing decisions, reducing the need for self-monitored blood glucose (SMBG) testing; CGM systems that do not require user calibrations will reduce this need even further. Training materials have improved to the point where self-training on the device is an effective alternative to one-on-one training for most patients, ${ }^{8}$ allowing clinicians more time to focus on diabetes management. Introducer needles are smaller and hidden, and sensor applicators have improved to make the insertion process simpler. ${ }^{9}$ Current-generation sensors last longer and resist acetaminophen interference. ${ }^{10}$ Some instances of skin irritation attributed to the adhesive pads have been addressed by manufacturing process changes, ${ }^{11}$ and long-term implantable CGM sensors, first available outside the United States, now provide an option for patients concerned with frequent device insertions and removals. Thus, many of the barriers for wider adoption of CGM have been addressed.

\section{Evidence and Implications}

A large and growing body of evidence supports the key messages of CGM and its use as a first-line option for insulinrequiring diabetes. Table 1 summarizes the real-world effectiveness of CGM and flash glucose monitoring in several large industry-sponsored observational studies, as well as a small, early study designed to gather normative data ${ }^{12}$ and a study of patients in Belgium ${ }^{13}$ who received funding for various CGM systems from the Belgian healthcare authority. Table 2 summarizes several major clinical trials designed to establish the efficacy of CGM and flash glucose monitoring in controlled settings.

In 2017, results of the Diamond ${ }^{14}$ and Gold ${ }^{15}$ randomized clinical trials were published simultaneously. Both studies were designed to compare CGM to usual care in people with T1D treated with multiple daily injections (MDI), and the Diamond study had a separately powered arm investigating

\section{Dexcom, Inc., San Diego, California.}

Data in this article have been presented at scientific conferences, including the 75th Scientific Sessions of the American Diabetes Association (Boston, MA, 5-9 June, 2015); the 76th Scientific Sessions of the American Diabetes Association (New Orleans, LA, 10-14 June, 2016); the 77th Scientific Sessions of the American Diabetes Association (San Diego, CA, 9-13 June, 2017); the 11th International Conference on Advanced Technologies \& Treatments for Diabetes (Vienna, Austria, 14-17 February, 2018); the 43rd Annual Conference of the International Society for Pediatric and Adolescent Diabetes (Innsbruck, Austria, 18-21 October, 2017); and the Diabetes UK Professional Conference (Manchester, England, 8-10 March, 2017). 
CGM in the context of MDI-treated T2D. ${ }^{16}$ Both studies enrolled subjects with above-target A1C values (Diamond, 7.5\%-9.9\%; Gold, $\geq 7.5 \%$ ), and both were designed to be relatively "low touch" with respect to encounter frequency.

In both studies, patients and clinicians were provided with a one-sheet set of general and patient-specific guidelines that had been shown earlier ${ }^{17}$ to improve glucose control in MDItreated patients with either T1D or T2D. General guidelines were to wear the CGM as much as possible; to look at the display device frequently; to maintain reasonable expectations; to approach alerts and alarms as helpful rather than punitive; to share the CGM data with others; and to learn from experience. Personalized guidelines involved establishing and using individual glucose targets; having a plan for preventing or responding to low glucose; knowing the plan for using CGM trends to improve prandial insulin dosing (remembering that an SMBG test is needed); and responding to high glucose levels between meals without stacking insulin. Adherence to these guidelines was not monitored in Diamond or Gold; however, they were felt to be understandable and easy to implement. ${ }^{17}$

\section{Wearing and checking the system}

In randomized controlled trials such as the JDRF CGM study ${ }^{4}$ and the STAR 3 study, ${ }^{18}$ sporadic wear of CGM was associated with sporadic A1C reductions, and wear times less than 6 days/week were associated with no A1C benefit in a separate study of youth with T1D. ${ }^{19}$ However, wearing the system is not enough; the data must be viewed and acted upon.

In examining data from 50,000 anonymized real-time CGM users, Parker et al. ${ }^{20}$ found a monotonic relationship between screen view frequency and time in range (TIR): the cohort of patients with the least-frequent screen views averaged $<2$ screen views per day and had $\sim 51 \%$ TIR $(70$ $180 \mathrm{mg} / \mathrm{dL}$ ), cohorts near the overall mean frequency of 9.1/ day had TIR near $60 \%$, and the cohort with the most-frequent screen views averaged $\sim 64 \%$ TIR. A similar analysis of data from more than 50,000 users of an intermittently scanned CGM system $^{21}$ found that TIR increased from $50 \%$ to $70 \%$ as screen view frequency increased from the lowest to highest frequency cohorts. In a separate analysis of data from four randomized clinical trials, subjects with TIR values near $60 \%$ had $\mathrm{A} 1 \mathrm{C}$ values in the $7.0 \%-7.5 \%$ range. $^{22}$ The increases in TIR were mostly attributable to decreases in hyperglycemia, rather than in hypoglycemia, suggesting that CGM use helps identify high glucose values that might otherwise remain undetected and undertreated.

\section{Maintaining reasonable expectations}

Realistic expectations help to avoid frustrations and disappointment for patients considering CGM, and CGM should not be imposed on those who are unwilling to use it consistently or incapable of using it beneficially. Patients should be advised on a basic troubleshooting checklist when their efforts aimed at good control fall short, and should be aware of the magnitude of expected discrepancies between SMBG and CGM values based on the system's performance characteristics. Blood glucose meters (BGMs), like CGM systems, are not perfect, and a significant proportion of cleared BGMs do not perform at the level for which they were cleared. ${ }^{23}$
Insulin-whether infused, injected, or inhaled - takes time to work; awareness of the delay between dose and effect is critical to avoid the temptation to stack insulin in response to stubborn or postprandial hyperglycemia. Similarly, the time required for a meal to raise glucose levels varies considerably based on its glycemic index ${ }^{24}$ and multiple other factors.

\section{Using alerts and trends}

If set correctly, the helpfulness of alerts persuades people to use them. Those that are too stringent or result from inaccurate CGM readings run the risk of going off too frequently and being viewed as a nuisance; conversely, alerts that are too lax may contribute to undertreatment of asymptomatic lows or highs. ${ }^{25}$ Arriving at appropriate alert settings is a customized process that depends on individual circumstances and goals, which are expected to change as the patient becomes more accustomed to the data and skilled in managing his or her diabetes. For example, patients with typically high glucose levels (e.g., those with A1Cs $>8 \%$ ) may find that the default high limit needs to be increased to perhaps $250 \mathrm{mg} / \mathrm{dL}{ }^{26}$ The recommended low alert level of $70 \mathrm{mg} / \mathrm{dL}$ generally gives the patient time to take action to prevent a clinical hypoglycemic episode and provides some margin for inaccurate readings, ${ }^{27}$ but a more conservative setting such as $80 \mathrm{mg} / \mathrm{dL}$ has been advocated to provide patients with more time to act. ${ }^{28}$ Hypoglycemia alerts $<80 \mathrm{mg} / \mathrm{dL}$ and hyperglycemia alerts $<200 \mathrm{mg} / \mathrm{dL}$ were both associated with lower mean sensor glucose values in a convenience sample of children and adolescents. ${ }^{29}$ A CGM app that allows for customized alert schedules during different times of the day and different days of the week is now available.

Systems may also be programmed to sound an alert in response to different rates of change. Rate-of-rise alerts may be especially useful for patients who occasionally forget to bolus before meals and rate-of-fall alerts may help patients with variable schedules avoid hypoglycemia. By default, rates of change are indicated on the CGM display as directional arrows and are intended to help patients respond to impending hyper- or hypoglycemia. Guidelines for using these trend arrows for fine-tuning insulin doses in children and adolescents ${ }^{30}$ and in adults ${ }^{31}$ are intended to balance complexity and utility in routine care. More sophisticated algorithms based on rates of change, threshold values, and time horizons form the basis of predictive alerts.

Severe hypoglycemia can be catastrophic; less severe hypoglycemia can impair the autonomic and symptomatic responses to later hypoglycemic episodes (the adage that "hypoglycemia begets hypoglycemia"). ${ }^{32}$ Trend arrows and alerts provided by real-time CGM systems play a key role in hypoglycemia avoidance strategies. A survey of regular ( $>6$ days/week) CGM users ${ }^{33}$ highlighted three key behaviors related to this goal. First, patients reduced or eliminated correction and mealtime bolus insulin doses in response to downward trending arrows. Second, patients responded to impending hypoglycemia with carbohydrates well before becoming symptomatic. Third and most consequentially, most survey respondents reported being awoken by low-glucose alerts at least once a week, enabling them to minimize or avoid nocturnal hypoglycemia.

Reductions in hypoglycemia are an important outcome in clinical trials. In the Diamond study, ${ }^{14}$ the median number of minutes per day with sensor glucose values $<50 \mathrm{mg} / \mathrm{dL}$ fell by 


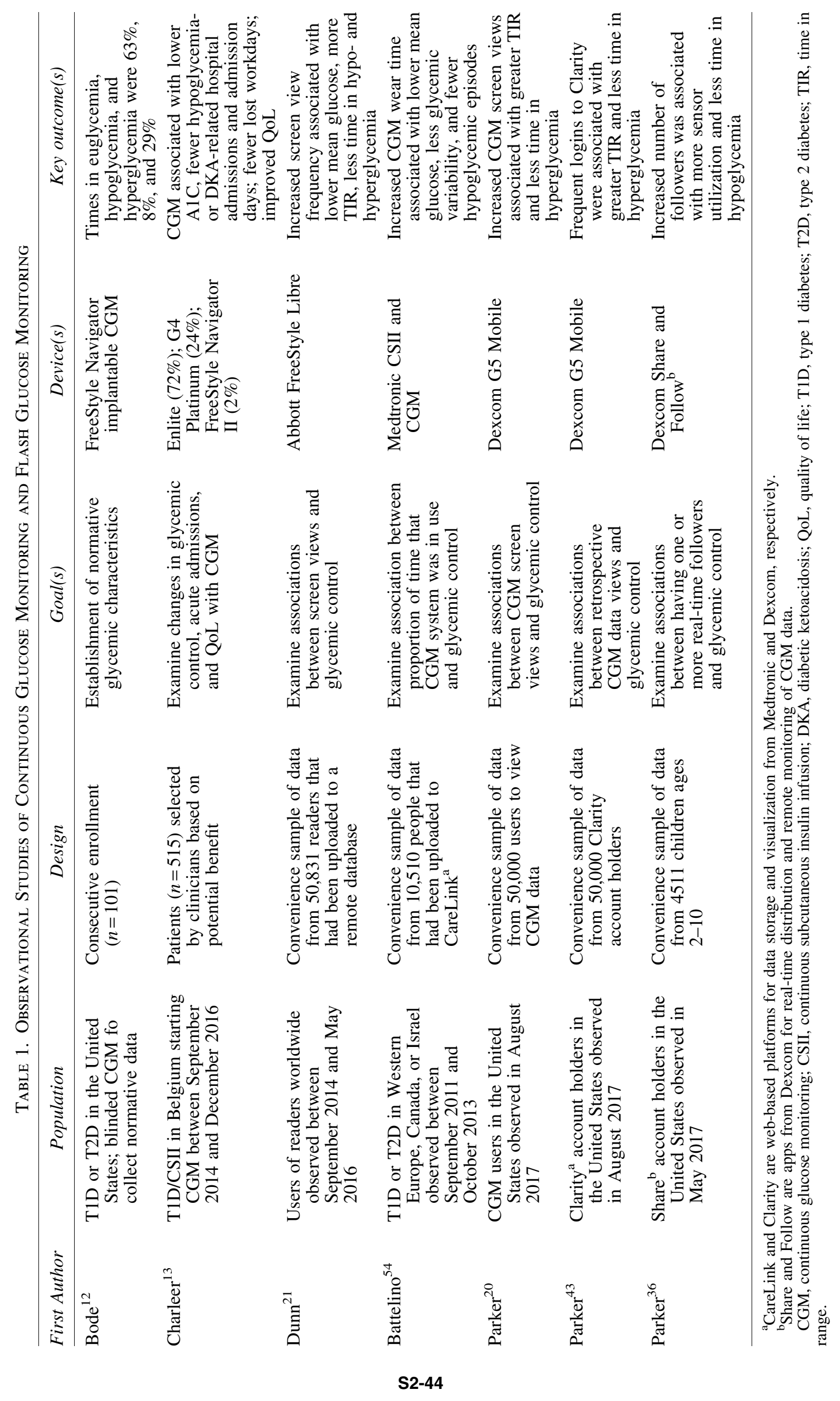




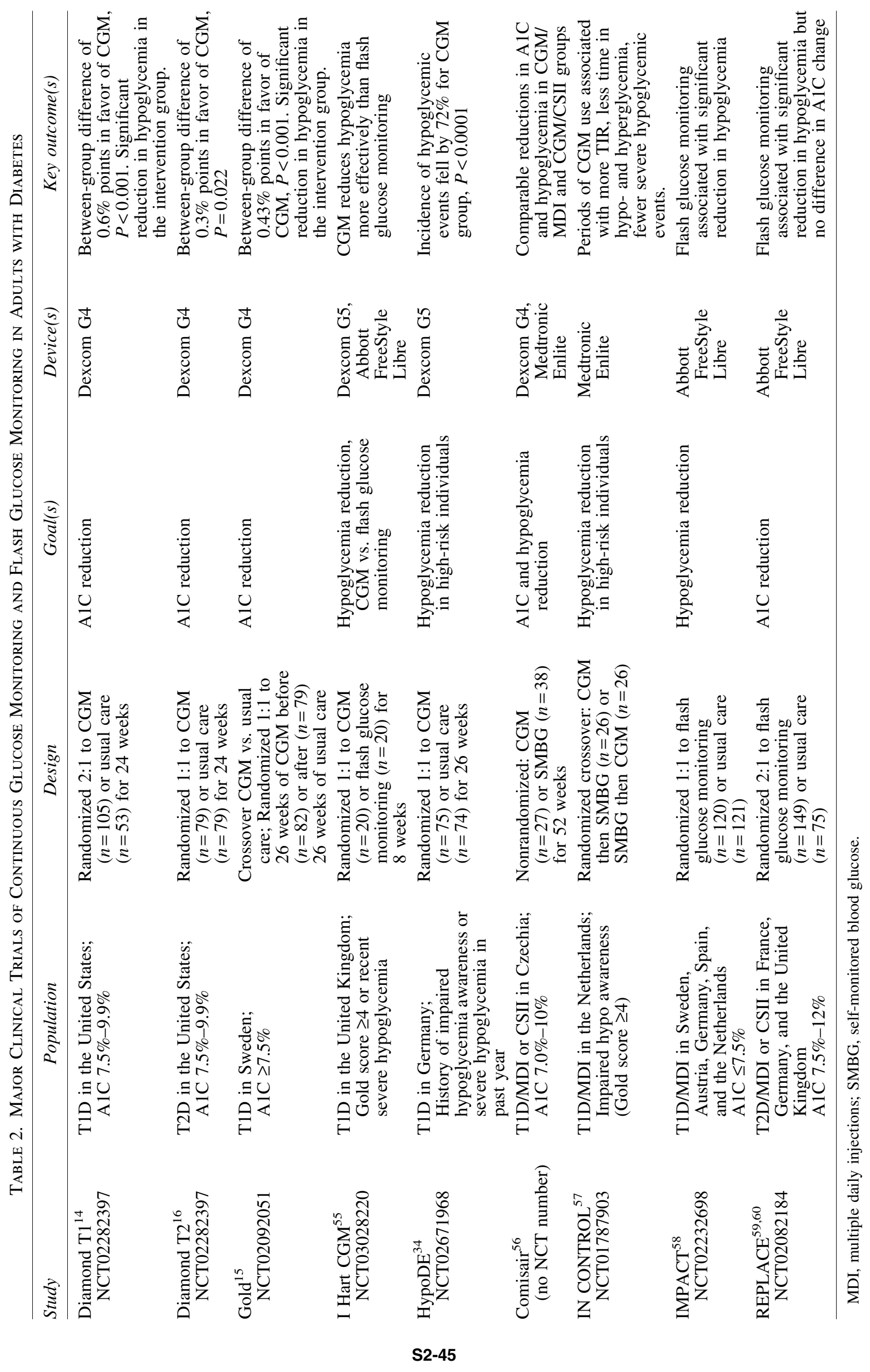


more than half (from 13 to 6 ) in the CGM group and rose from 18 to 20 in the control group $(P<0.001)$. More recently, the HypoDE study ${ }^{34}$ aimed to quantify the effect of CGM usage among hypoglycemia-prone individuals (evidenced by a history of impaired hypoglycemia awareness or severe hypoglycemia during the previous year) with T1D treated with MDI. In this study, the mean number of hypoglycemic events per 28 days among participants in the CGM group was reduced from 10.8 to 3.5; reductions among control participants were negligible (from 14.4 to 13.7). The relative contribution of daytime and nocturnal alerts, trend arrows, or unprompted screen views to these favorable reductions is a topic for further study. The availability of alerts and alarms in real time but not intermittently scanned CGM systems should play a role in selecting the CGM type that is most appropriate for each patient. ${ }^{35}$

\section{Real-time sharing}

Sharing of CGM data allows outsourcing of some shortterm diabetes management decisions and provides patients with more opportunities for collaborative pattern discovery and shared decision-making. As an example, Dexcom's "Share" and "Follow" mobile applications were designed to allow CGM data from a single "Sharer" to be distributed in real time to up to five "Followers" running the corresponding apps. Sharing is especially useful for patients who are unable to fully manage their own disease, such as young children; following is especially useful for caregivers who can intervene in a timely manner, such as parents of young children. In an exploratory analysis of Sharers ages $2-10$, those with no paired Followers had $16 \%$ of their sensor glucose values in the $<70 \mathrm{mg} / \mathrm{dL}$ range, whereas children with five Followers had only $8.7 \%$ of their values in this range. ${ }^{36}$ A more modest $14 \%$ reduction in the percentage of sensor glucose values $<70 \mathrm{mg} / \mathrm{dL}$ was found in a similar analysis of adults 65 years and older with at least one Follower. ${ }^{37}$ Among adolescents, the effects of sharing CGM data are more variable and depend on the context of family dynamics: at the extremes, the technology can be viewed either as a trusted best friend contributing to independent decision-making or as an unwelcome parental spy. ${ }^{38}$

Patients are often advised to fold CGM reviews into their daily routines and build narratives around their glucose levels and activities. In an analysis of adults in the JDRF CGM trial, Ritholz et al. 39 characterized "Responders" as those with baseline $\mathrm{A} 1 \mathrm{Cs} \geq 7 \%$ and $\mathrm{A} 1 \mathrm{C}$ reductions $\geq 0.5 \%$ points or those whose time $<70 \mathrm{mg} / \mathrm{dL}$ decreased while maintaining A1Cs $<7 \%$. In either case, Responders gave credit to retrospective pattern analysis of their CGM data and to "significant other" or spousal involvement for the effective use of CGM.

\section{Retrospective reviewing}

While CGM data gathered in real time can guide in-themoment management decisions, it can also inform longer term decisions related to patterns identified through retrospective data analysis. Software for CGM data visualization ranges from general purpose (Excel, Tableau) to manufacturer-specific webbased options such as CareLink ${ }^{\circledR}$ (Medtronic), Clarity ${ }^{\circledR}$ (Dexcom), and LibreView ${ }^{\mathrm{TM}}$ (Abbott). The ambulatory glucose profile $^{40}$ is a device-agnostic, single-page report that is designed for rapid, easy interpretation of SMBG and/or CGM data. Its standardized reports have recently been shown to save time in the context of clinic visits, translating into more time for shared decision-making and education. ${ }^{41}$

Data from Dexcom Clarity suite of mobile and web-based software for retrospectively sharing and analyzing CGM data confirm the benefits of these behaviors: in a recent analysis of 50,000 users of the Dexcom G5 Mobile app, patients who logged in four or more times to Clarity during August 2017 had significantly more TIR, lower mean sensor glucose values, and less time in hyperglycemia than patients who did not log in during this interval. ${ }^{42}$ A recent update allows Clarity users to opt in to weekly push notifications and/or e-mail messages that include summary statistics, patterns, and comparisons to the week before. Opting in to this service lowers the effort required to obtain the reports and reframes weekly retrospective reviews as the default behavior.

\section{SMBG testing and quality of life}

On December 20, 2016, the U.S. Food and Drug Administration (FDA) approved labeling for the Dexcom G5 Mobile CGM System for making diabetes treatment decisions without the need for a confirmatory blood glucose measurement. ${ }^{43}$ This decision was at odds with a minority viewpoint suggesting that CGM data might suffice for some diabetes treatment decisions (such as carbohydrate ingestion, exercise, meal timing, and sleep), but were not accurate enough for dosing insulin. ${ }^{44}$ Three weeks later, the System was reclassified by the Centers for Medicare and Medicaid Services as durable medical equipment able to replace BGMs. ${ }^{45}$

Given the shortcomings of SMBG testing, ${ }^{46}$ these decisions were welcomed by the community and were seen as legitimizing the reported behavior of many participants in the T1D Exchange Registry with respect to "dosing off the CGM.",47 The quantity and quality of CGM data-hundreds of point estimates per day, trends, and alarms-far exceed that provided by home BGMs. The safety of CGM-based dosing decisions was confirmed in the REPLACE-BG study. ${ }^{48}$

With the FDA decision allowing nonadjunctive use, the required number of SMBG tests decreased for most CGM users in the United States, but periodic SMBG tests are still required to calibrate most CGM systems. Recent advances in sensors and algorithms have resulted in CGM systems that are calibrated during the manufacturing process and are stable enough so as to not require calibrations during use, further reducing the need for SMBG testing. SMBG results will remain useful when CGM data are unavailable (e.g., during sensor warm-ups) or when there are mismatches between the CGM data and patient's expectations or symptoms. Users of the FreeStyle Libre flash glucose monitoring system (Abbott) are also advised to perform an SMBG test when experiencing potential hypoglycemia or hyperglycemia; when the system's glucose values are changing by more than $2 \mathrm{mg} /(\mathrm{dL} \cdot \mathrm{min})$; when the system's display does not include a current glucose number or trend arrow; or to confirm system-reported hypoglycemia or impending hypoglycemia.

Reductions in hypoglycemia, fewer SMBG tests, and fruitful engagement of others in diabetes management decisions may all contribute to improved quality-of-life (QoL) outcomes for people using CGM. In the Diamond study, participants completed several questionnaires related to QoL. ${ }^{49}$ Compared with subjects in the control group, those in the CGM group had greater increases in hypoglycemic confidence and greater decreases in 
diabetes distress. A separate observational cohort study of 515 adults with T1D on continuous subcutaneous insulin infusion (CSII) therapy (the RESCUE trial ${ }^{13}$ ) was prompted by the Belgian health authority's decision to reimburse real-time CGM systems for $25 \%$ of CSII users; specialized centers across Belgium were allowed to decide which patients would receive reimbursement for their systems. After at least a 12-month use of real-time CGM, subjects had improved glycemic control, lower risk of acute diabetes-related complications, and higher QoL attributed to less fear of hypoglycemia. CGM use was also associated with reduced rates of hypoglycemia-related hospitalizations and less work absenteeism, which could offset some of the costs of this technology.

\section{Prospects for the Near Term}

The next few years for CGM systems will hold challenges and opportunities. Because CGM is a young technology and still undergoing rapid and sometimes abrupt changes, interpretation of longitudinal data is challenging because early conclusions were often reached with now-obsolete hardware and software. Compared with recommendations based on SMBG test results, recommendations for lifestyle and insulin dosage changes based on the direction and degree of CGM trend data are more nuanced and variable. ${ }^{50}$ Although some systems no longer require a dedicated receiver and some sensors are implantable, all real-time systems require a display device and a skin-adherent patch. Although the amount of numerical data can be intimidating to some patients, beneficial A1C reductions in the Diamond study did not depend on educational attainment, numeracy scores, or age. Clinical inertia in primary care settings, ${ }^{51}$ out-of-pocket costs, and the perceived need for extensive specialized training remain barriers to wider adoption of CGM.

On March 27, 2018, the FDA created a new category of "integrated continuous glucose monitoring (iCGM)" systems. The Dexcom G6 ${ }^{\circledR}$ CGM System was the first device in this category and is subject to special controls for class II that provide assurances of its safety and effectiveness. Importantly, the new classification will allow for more rapid innovation, as some changes that would have required FDA premarket approval as a class III device may now be reviewed by FDA in less time. Certain changes may even be implemented without prior FDA review once all quality system and regulatory documentation requirements are fulfilled. The G6 system is indicated to replace fingerstick blood glucose testing for diabetes treatment decisions and to autonomously communicate with digitally connected devices, including automated insulin delivery systems.

The low penetration of CGM among MDI-using patients implies a large number of people who have yet to try it. The latest generation of systems promises improved accuracy, durability, ease of use, and comfort. Importantly, CGM data are easily integrated with mobile health apps that provide users with information from other sources related to insulin delivery, food, and activity. Thanks to the interoperability of the G6 System, CGM data may soon be incorporated into smart insulin pen systems that track insulin on board and provide dosing recommendations. CGM is also an emerging area of investigation for reduction of hypoglycemia and severe hypoglycemia in hospitalized patients, ${ }^{52}$ which are associated with poor outcomes. Finally and as discussed elsewhere in this supplement, CGM is an essential component of artificial pancreas systems.

\section{Summary}

CGM is a representative case study in patient engagement and participatory medicine. It illustrates how technological developments and patient advocacy can combine to produce a breakthrough option for millions of people who depend on insulin. It enables patients to make better-informed decisions regarding diet, exercise, and insulin dose adjustments, improves the quality of glycemic control, reduces exposure to hyper- and hypoglycemia, and facilitates attainment of lower target $\mathrm{A} 1 \mathrm{C}$ and average glucose levels. CGM use allows for fewer SMBG tests and improves the QoL, regardless of insulin delivery method. When shared with other individuals, the information provided by CGM systems contributes to active and collaborative decision-making. Thanks to recent improvements in algorithms and devices and to the large and growing evidence base in its favor, CGM adoption is increasing. Until a "perfect" risk-free insulin ${ }^{53}$ or a viable strategy for pancreatic beta-cell replacement is found, CGM will remain an essential technology and should be viewed as a first-line option and the standard of care in treating type 1 and insulin-requiring type 2 diabetes.

\section{Author Disclosure Statement}

J.B.W. is an employee of and has a financial interest in Dexcom, Inc. J.B.W. thanks Drs. Andrew Parker, David Price, Sarah Puhr, and Tomas Walker for discussions on the topic of CGM.

\section{References}

1. Lee V, Thurston T, Thurston C: A comparison of discovered regularities in blood glucose readings across two data collection approaches used with a type 1 diabetic youth. Methods Inf Med 2017;56:e84-e91.

2. Gimenez M, Tannen AJ, Reddy M, et al.: Revisiting the relationships between measures of glycemic control and hypoglycemia in continuous glucose monitoring data sets. Diabetes Care 2018;41:326-332.

3. van Beers CAJ, Caris MG, DeVries JH, Serne EH: The relation between $\mathrm{HbAlc}$ and hypoglycemia revisited; a secondary analysis from an intervention trial in patients with type 1 diabetes and impaired awareness of hypoglycemia. J Diabetes Complications 2018;32:100-103.

4. Juvenile Diabetes Research Foundation Continuous Glucose Monitoring Study Group, Tamborlane WV, Beck RW, et al:: Continuous glucose monitoring and intensive treatment of type 1 diabetes. N Engl J Med 2008;359:14641476.

5. Facchinetti A: Continuous glucose monitoring sensors: past, present and future algorithmic challenges. Sensors (Basel) 2016;16:E2093.

6. Miller K, Foster N, DeSalvo D, et al.: Continuous glucose monitoring (CGM) use in type 1 diabetes: an update from the T1D exchange clinic registry. Pediatric Diabetes 2016; 17(S24):49.

7. Foster NC, Miller KM, DiMeglio LA, et al.: Marked increases in CGM use has not prevented increases in HbA1c levels in participants in the T1D Exchange (T1DX) Clinic Network. Diabetes 2018;67. In press. 
8. Dunn L, Chen A, Clukey R, Leone KJ: Comparing effectiveness of self vs. one-on-one training on use of the Dexcom G5 Mobile continuous glucose monitoring system. Diabetes 2017;66:A184.

9. Clukey R, North B, Parkin C: Utilization of human factors analysis throughout product development enhances usability of CGM sensor applicators. Diabetes 2017;66:A184.

10. Calhoun P, Johnson TK, Hughes J, et al.: Resistance to acetaminophen interference in a novel continuous glucose monitoring system. J Diabetes Sci Technol 2018;12:393396.

11. Gisin V, Chan A, Welsh JB: Manufacturing process changes and reduced skin irritations of an adhesive patch used for continuous glucose monitoring devices. J Diabetes Sci Technol 2018;12:725-726.

12. Bode BW, Schwartz S, Stubbs HA, Block JE: Glycemic characteristics in continuously monitored patients with type 1 and type 2 diabetes: normative values. Diabetes Care 2005;28:2361-2366.

13. Charleer S, Mathieu C, Nobels F, et al.: Effect of continuous glucose monitoring on glycemic control, acute admissions, and quality of life: a real-world study. J Clin Endocrinol Metab 2018;103:1224-1232.

14. Beck RW, Riddlesworth T, Ruedy K, et al.: Effect of continuous glucose monitoring on glycemic control in adults with type 1 diabetes using insulin injections: the DIAMOND randomized clinical trial. JAMA 2017;317: 371-378.

15. Lind M, Polonsky W, Hirsch IB, et al.: Continuous glucose monitoring vs. conventional therapy for glycemic control in adults with type 1 diabetes treated with multiple daily insulin injections: the GOLD randomized clinical trial. JAMA 2017;317:379-387.

16. Beck RW, Riddlesworth TD, Ruedy K, et al.: Continuous glucose monitoring versus usual care in patients with type 2 diabetes receiving multiple daily insulin injections: a randomized trial. Ann Intern Med 2017;167:365-374.

17. Price DA, Gerety G, Blevins TC, Casal E: Use of continuous glucose monitoring (CGM) with personalized instructions provides glycemic benefit to patients using multiple daily insulin injections (MDI). Diabetes 2015;64: A195.

18. Bergenstal RM, Tamborlane WV, Ahmann A, et al.: Effectiveness of sensor-augmented insulin-pump therapy in type 1 diabetes. N Engl J Med 2010;363:311-320.

19. Giani E, Snelgrove R, Volkening LK, Laffel LM: Continuous glucose monitoring (CGM) adherence in youth with type 1 diabetes: associations with biomedical and psychosocial variables. J Diabetes Sci Technol 2017;11: 476-483.

20. Parker AS, Welsh JB, Dunn LJ, et al.: Insights from big data (1): viewing of real-time continuous glucose monitoring data and its impact on time in range. Diabetes Technol Ther 2018; 20:A-121.

21. Dunn TC, Xu Y, Hayter G, Ajjan RA: Real-world flash glucose monitoring patterns and associations between selfmonitoring frequency and glycaemic measures: a European analysis of over 60 million glucose tests. Diab Res Clin Pract 2018;137:37-46.

22. Calhoun P, Johnson T, Welsh J, et al.: Associations between A1C and continuous glucose monitoring-derived glycemic parameters. Diabetes 2018;67. In press.

23. Klonoff DC, Prahalad P: Performance of cleared blood glucose monitors. J Diabetes Sci Technol 2015;9:895-910.
24. Atkinson FS, Foster-Powell K, Brand-Miller JC: International tables of glycemic index and glycemic load values: 2008. Diabetes Care 2008;31:2281-2283.

25. Mastrototaro J, Welsh JB, Lee S: Practical considerations in the use of real-time continuous glucose monitoring alerts. J Diabetes Sci Technol 2010;4:733-739.

26. Hirsch IB: Clinical review: realistic expectations and practical use of continuous glucose monitoring for the endocrinologist. J Clin Endocrinol Metab 2009;94:2232-2238.

27. Cryer PE: Preventing hypoglycaemia: what is the appropriate glucose alert value? Diabetologia 2009;52:35-37.

28. Peyser TA, Nakamura K, Price D, et al.: Hypoglycemic accuracy and improved low glucose alerts of the latest Dexcom G4 Platinum continuous glucose monitoring system. Diabetes Technol Ther 2015;17:548-554.

29. Welsh JB, Walker T, Liu X: Continuous glucose monitoring screen views and alert settings affect glycemic control in children and adolescents. Diabet Med 2017;34:129.

30. Laffel LM, Aleppo G, Buckingham BA, et al.: A practical approach to using trend arrows on the Dexcom G5 CGM system to manage children and adolescents with diabetes. J Endocr Soc 2017;1:1461-1476.

31. Aleppo G, Laffel LM, Ahmann AJ, et al.: A practical approach to using trend arrows on the Dexcom G5 CGM system for the management of adults with diabetes. J Endo Soc 2017;1:1445-1460.

32. Cryer PE: Hypoglycemia begets hypoglycemia in IDDM. Diabetes 1993;42:1691-1693.

33. Pettus J, Price DA, Edelman SV: How patients with type 1 diabetes translate continuous glucose monitoring data into diabetes management decisions. Endocr Pract 2015;21: 613-620.

34. Heinemann L, Freckmann G, Ehrmann D, et al.: Real-time continuous glucose monitoring in adults with type 1 diabetes and impaired hypoglycaemia awareness or severe hypoglycaemia treated with multiple daily insulin injections (HypoDE): a multicentre, randomised controlled trial. Lancet 2018;391:1367-1377.

35. Adolfsson P, Parkin CG, Thomas A, Krinelke LG: Selecting the appropriate continuous glucose monitoring system-a practical approach. Eur Endocrinol 2018;14:24-29.

36. Parker A, Welsh J, Jimenez A, Graham C: Effects of sharing continuous glucose monitoring (CGM) data from young children with diabetes on CGM usage and hypoglycemic exposure. Pediatr Diabetes 2017;18:76-77.

37. Parker AS, Jimenez A, Welsh JB, et al.: Hypoglycemic exposure among older adults using the Dexcom Share Cloud. J Diabetes Sci Technol 2017;12:A60.

38. Messer LH, Johnson R, Driscoll KA, Jones J: Best friend or spy: a qualitative meta-synthesis on the impact of continuous glucose monitoring on life with type 1 diabetes. Diabet Med 2018;35:409-418.

39. Ritholz MD, Atakov-Castillo A, Beste M, et al.: Psychosocial factors associated with use of continuous glucose monitoring. Diabet Med 2010;27:1060-1065.

40. Mazze RS, Lucido D, Langer O, et al.: Ambulatory glucose profile: representation of verified self-monitored blood glucose data. Diabetes Care 1987;10:111-117.

41. Mullen DM, Bergenstal R, Criego A, et al.: Time savings using a standardized glucose reporting system and ambulatory glucose profile. J Diabetes Sci Technol 2018;12:614621.

42. Parker AS, Welsh J, Jimenez A, Walker T: Insights from big data (2): benefits of self-guided retrospective review of 
continuous glucose monitoring reports. Diabetes Technol Ther 2018;20:A-27.

43. PMA Supplement Approval Letter, P120005/S041 for Dexcom G5 Mobile Continuous Glucose Monitoring System. 2016. www.accessdata.fda.gov/cdrh_docs/pdf12/P120005S0 41a.pdf (accessed May 20, 2018).

44. Shapiro AR: FDA approval of nonadjunctive use of continuous glucose monitors for insulin dosing: a potentially risky decision. JAMA 2017;318:1541-1542.

45. Centers for Medicare and Medicaid Services: Ruling No.: [CMS-1682-R].,2017. www.cms.gov/Regulations-andGuidance/Guidance/Rulings/Downloads/CMS1682R.pdf (accessed May 20, 2018).

46. Price $\mathrm{D}$, Walker $\mathrm{T}$ : The rationale for continuous glucose monitoring-based diabetes treatment decisions and nonadjunctive continuous glucose monitoring use. Eur Endocrinol 2016;12:24-30.

47. Wong JC, Foster NC, Maahs DM, et al.: Real-time continuous glucose monitoring among participants in the T1D Exchange clinic registry. Diabetes Care 2014;37:27022709.

48. Aleppo G, Ruedy KJ, Riddlesworth TD, et al.: REPLACEBG: a randomized trial comparing continuous glucose monitoring with and without routine blood glucose monitoring in adults with well-controlled type 1 diabetes. Diabetes Care 2017;40:538-545.

49. Polonsky WH, Hessler D, Ruedy KJ, et al.: The impact of continuous glucose monitoring on markers of quality of life in adults with type 1 diabetes: further findings from the DIAMOND randomized clinical trial. Diabetes Care 2017; 40:736-741.

50. Davidson MB: Continuous glucose monitoring in patients with type 1 diabetes taking insulin injections. JAMA 2017; 317:363-364.

51. Ziemer DC, Miller CD, Rhee MK, et al.: Clinical inertia contributes to poor diabetes control in a primary care setting. Diabetes Educ 2005;31:564-571.

52. Cruz P, Blackburn MC, Tobin GS: A systematic approach for the prevention and reduction of hypoglycemia in hospitalized patients. Curr Diab Rep 2017;17:117.
53. Zaykov AN, Mayer JP, DiMarchi RD: Pursuit of a perfect insulin. Nat Rev Drug Discov 2016;15:425-439.

54. Battelino T, Liabat S, Veeze HJ, et al.: Routine use of continuous glucose monitoring in 10501 people with diabetes mellitus. Diabet Med 2015;32:1568-1574.

55. Reddy M, Jugnee N, El Laboudi A, et al.: A randomized controlled pilot study of continuous glucose monitoring and flash glucose monitoring in people with type 1 diabetes and impaired awareness of hypoglycaemia. Diabet Med 2018; 35:483-490.

56. Šoupal J, Petruželková L, Flekač M, et al.: Comparison of different treatment modalities for type 1 diabetes, including sensor-augmented insulin regimens, in 52 weeks of followup: A COMISAIR Study. Diabetes Technol Ther 2016;18: 532-538.

57. van Beers CA, DeVries JH, Kleijer SJ, et al.: Continuous glucose monitoring for patients with type 1 diabetes and impaired awareness of hypoglycaemia (IN CONTROL): a randomised, open-label, crossover trial. Lancet Diabetes Endocrinol 2016;4:893-902.

58. Bolinder J, Antuna R, Geelhoed-Duijvestijn P, et al.: Novel glucose-sensing technology and hypoglycaemia in type 1 diabetes: a multicentre, non-masked, randomised controlled trial. Lancet 2016;388:2254-2263.

59. Haak T, Hanaire H, Ajjan R, et al.: Use of flash glucosesensing technology for 12 months as a replacement for blood glucose monitoring in insulin-treated type 2 diabetes. Diabetes Ther 2017;8:573-586.

60. Haak T, Hanaire H, Ajjan R, et al.: Flash glucose-sensing technology as a replacement for blood glucose monitoring for the management of insulin-treated type 2 diabetes: a multicenter, open-label randomized controlled trial. Diabetes Ther 2017;8:55-73.

Address correspondence to: John B. Welsh, MD, PhD

Dexcom, Inc. 6340 Sequence Drive San Diego, CA 92121

E-mail: john.welsh@dexcom.com 